\title{
APRENDIZAJE BASADO EN
} PROYECTOS MEDIADOS POR TIC PARA SUPERAR DIFICULTADES EN EL APRENDIZAJE DE OPERACIONES BÁSICAS MATEMÁTICAS

\section{LEARNING BASED ON PROJECTS MEDIATED TIC TO OVERCOME DIFFICULTIES IN LEARNING BASIC MATHEMATICS OPRATIONS}

\section{Nury Andrea Vargas Vargas ${ }^{1}$}

Jorge Armando Niño Vega ${ }^{2}$

Flavio Humberto Fernández Morales ${ }^{3}$

Universidad Pedagógica y Tecnológica de Colombia, Duitama, Colombia.

1 Docente, Licenciada en Tecnología, estudiante de Maestría en TIC Aplicadas a las Ciencias de la Educación, Universidad Pedagógica y Tecnológica de Colombia, Duitama, Colombia. E-mail: nury.a.vargas@gmail.com |ORCID ID: https://orcid. org/0000-0002-6004-8482

2 Docente investigador, Licenciado en Tecnología, Magíster en TIC Aplicadas a las Ciencias de la Educación, Universidad Pedagógica y Tecnológica de Colombia, Duitama, Colombia. E-mail: Jorge.ninovega@gmail.com IORCID ID: https://orcid. org/0000-0001-7803-5535

3 Docente investigador, Ingeniero Electrónico, Doctor en Ingeniería Electrónica, Universidad Pedagógica y Tecnológica de Colombia, Duitama, Colombia. E-mail: flaviofm1@gmail.com |ORCID ID: https://orcid.org/0000-0002-8970-7146 


\section{RESUMEN}

En el documento se presenta una aplicación del Aprendizaje Basado en Proyectos (ABP), mediado por Tecnologías de la Información y la Comunicación (TIC), para superar las Dificultades de Aprendizaje Matemático (DAM), centradas en las operaciones básicas. La población fue de 23 estudiantes del grado sexto, de una institución educativa pública colombiana, con dificultades de aprendizaje en tres competencias: conceptual, razonamiento lógico y solución de operaciones. La investigación es de carácter mixto, con aplicación de una prueba inicial y otra final para establecer el cambio en las competencias matemáticas. El grupo se dividió en dos: uno construyó los proyectos a partir de un Material Educativo Computarizado (MEC), mientras que el otro lo hizo a partir de folletos escritos. La prueba inicial arrojó un $27 \%$ de estudiantes en desempeño bajo, y $7 \%$ en superior; mientras que en la prueba final, el $83 \%$ de los estudiantes se ubicó en superior y ninguno en bajo. Se concluye que el ABP y las TIC, son una excelente estrategia de aprendizaje para la adquisición de conocimientos matemáticos, en estudiantes con dificultades de aprendizaje.

\section{PALABRAS CLAVE:}

Aprendizaje basado en proyectos, Operaciones Básicas matemáticas, tecnologías de la información y la comunicación, enseñanza de MATEMÁTICAS.

\section{ABSTRACT}

The document presents an application of Project-Based Learning (PBL), mediated by Information and Communication Technologies (ICT), to overcome Mathematical Learning Difficulties (MLD), focused on basic operations. The population was 23 students of the sixth grade, from a Colombian public educational institution, with learning difficulties in three competences: conceptual, logical reasoning and operations solution. The research is mixed in nature, with the application of an initial and a final test to establish the change in mathematical competences. The group was divided into two: one built the projects from a Computerized Educational Material (CEM), while the other did it from written brochures. The initial test showed $27 \%$ of students in low performance, and $7 \%$ in higher; while in the final test, $83 \%$ of the students were in superior performance and none in low. It is concluded that PBL and ICT are an excellent learning strategy for the acquisition of mathematical knowledge, in students with learning difficulties.

\section{KEYWORDS}

Project-based learning, Mathematical basic operations, Information and Communication Technologies, Mathematical teaching.

\section{INTRODUCCIÓN}

La enseñanza de las Operaciones Básicas Matemáticas (OBM), hace parte de la alfabetización ciudadana, pues se trata de conocimientos que toda persona utiliza en sus actividades diarias (Aldana-Bermúdez \& LópezMesa, 2016). Sin embargo, los estudiantes con frecuencia presentan dificultades en el manejo de conceptos y poca comprensión en temas que son básicos para avanzar sistemáticamente en su formación académica (Hernández-Suárez, Prada-Núñez \& Gamboa-Suárez, 2017). Ejemplo de ello son las dificultades en la comprensión y dominio conceptual de las cuatro operaciones básicas: adición, sustracción, multiplicación y división, que son esenciales para avanzar en temáticas de grados superiores (Botero, Rentería \& Vergara, 2016).

En este sentido, los estudiantes requieren métodos de aprendizaje diferentes a los tradicionales, que les permitan comprender mejor los conceptos y reflejarlos en la realidad 
(Gutiérrez-Rodríguez, 2018; VesgaBravo \& Escobar-Sánchez, 2018). Estos cambios requieren de una continua transformación en el material didáctico utilizado en el proceso de enseñanza-aprendizaje (OrdóñezOrtega, Gualdrón-Pinto \& AmayaFranky, 2019). No obstante, para que el estudiante desarrolle competencias de aprendizaje modernas, los docentes también deben actualizar las técnicas de enseñanza (Morales-Bueno, 2018). Esto implica la necesidad de adoptar estrategias pedagógicas que generen un aprendizaje significativo y colaborativo en los estudiantes para resolver problemas cotidianos (AlvisPuentes, Aldana-Bermúdez \& CaicedoZambrano,2019).

Una estrategia pedagógica interesante es el Aprendizaje Basado en Proyectos (ABP), que permite a los docentes orientar el proceso formativo con herramientas innovadoras, a la vez que realza el papel de los estudiantes como sujetos activos de dicho proceso (GarcíaMartín \& Pérez-Martínez, 2018). El ABP busca incluir en las clases la elaboración de proyectos que integran la teoría, la práctica y el trabajo cooperativo (Gutiérrez-Rico, AlmarazRodríguez \& Bocanegra-Vergara, 2019). Estas construcciones demuestran la aplicabilidad de conocimiento en las diferentes disciplinas, apropiados a través de un proceso formativo que aprovecha la oportunidad para verificar, confrontar y socializar lo que se obtiene por medio de la observación y la experiencia individual (Fernández \& Duarte, 2013; GarzónSaladen \& Romero-González, 2018).

El aprendizaje se da a partir de la interacción entre el objeto de aprendizaje y el sujeto que aprende, teniendo como principal intermediario al docente, que es el que motiva, lidera y orienta a partir de una planeación pertinente y organizada, con la cual ejecuta lo que pretende enseñar (Trujillo-Lozada, Hurtado-Zúñiga \& Pérez-Paredes, 2019; Fernández-Guerrero \& González-Ferro, 2017). En este sentido, es interesante explorar las Tecnologías de Información y Comunicación (TIC), como un mediador y gestor del conocimiento (MartínezLópez \& Gualdrón-Pinto, 2018). Esto debido a que, en la actualidad, los estudiantes tienden a ser autodidactas y su fuente principal de información proviene de datos que se encuentran en espacios digitales de fácil acceso (BuitragoGuzmán, 2014; Revelo-Rosero, Lozano \& Bastidas-Romo, 2019).

Las TIC han impactado a las diversas áreas de la actividad humana, como: medicina, ingeniería y administración, por mencionar algunas (Marino-Vera, Mendoza \& GualdrónGuerrero, 2017; Vergara-Arrieta, 2015; Cáceres \& Amaya, 2016). En el caso de la educación, las nuevas tecnologías han favorecido la aparición, entre otras, de herramientas como: Ambientes Virtuales de Aprendizaje (AVA), Materiales Educativos Computarizados (MEC), Objetos Virtuales de Aprendizaje (OVA), así como plataformas para la administración de contenidos y páginas web especializadas (Niño-Vega et al., 2017; Ruiz-Macías \& Duarte, 2018; SalcedoRamírez, Fernández Morales \& Duarte, 2017; Cruz-Rojas, Molina-Blandón \& Valdiri-Vinasco, 2019; Barrera-Mesa, Fernández-Morales \& Duarte, 2017). Lo anterior hace interesante explorar la utilización de estas tecnologías para el aprendizaje de las OBM, especialmente en estudiantes que presentan dificultades para su aplicación.

En este documento se presenta una aplicación del ABP, mediado por TIC, para superar las Dificultades de Aprendizaje Matemático (DAM), centradas en las operaciones básicas. Se trabajó con estudiantes del grado sexto, de una institución educativa pública colombiana, con dificultades de aprendizaje en tres competencias: 
conceptual, razonamiento lógico y solución de operaciones. A continuación, se describe la metodología empleada en la ejecución de esta investigación. Luego se describe la experiencia de aula, junto con el análisis de las pruebas realizadas. Finalmente, se brindan las conclusiones obtenidas a partir de la reflexión sobre la experiencia.

\section{MARCO TEÓRICO Y MÉTODO}

\subsection{Aprendizaje Basado en Proyectos}

El ABP es una experiencia de aprendizaje que involucra al estudiante en un proyecto complejo y significativo, el cual permite el desarrollo integral de sus capacidades, habilidades, actitudes y valores (Morales-Bueno, 2018). El ABP también se presenta como una alternativa eficaz para responder a desafíos de aprendizaje, convirtiéndose en una metodología clave en la preparación integral, crítica e investigadora del estudiante (Gómez-Pablos, 2018). En este sentido, el ABP en la escuela es la mejor manera de utilizar el potencial innato del alumnado, y de prepararlos para ser ciudadanos responsables y motivados hacia el aprendizaje (VegaMonsalve \& Ruiz-Restrepo, 2018). Por ello, las experiencias en el aprendizaje que implican la innovación en proyectos multidisciplinares, posibilitan al estudiante el trabajo de diferentes conceptos y áreas de conocimiento.

La estimulación que se recibe con esta estrategia, permite que los estudiantes se sientan motivados y comprometidos en desarrollar sus actividades y fortalecer de manera autónoma su aprendizaje (Villamil-Rincón, Aldana-Bermúdez \& Wagner-Osorio, 2018). Malpartida-Márquez (2018), sustenta como objetivos principales en el ABP: fomentar el trabajo en equipo y colaborativo, desarrollo de capacidades, habilidades y valores, genera un entorno motivador, desarrolla el auto aprendizaje y el pensamiento creativo, fomenta la indagación de los estudiantes y contribuye con los procesos de aprendizaje. Estos objetivos conllevan a una educación centrada en la enseñanza, donde la construcción del conocimiento se da a través de la realidad.

En la tabla 1 se enlistan los proyectos a ser desarrollados por los estudiantes, a fin de superar sus dificultades con las Operaciones Básicas Matemáticas (OBM).

Tabla 1. Proyectos para desarrollar las OBM

\begin{tabular}{|l|l|}
\hline \multicolumn{1}{|c|}{ Nombre } & \multicolumn{1}{c|}{ Descripción } \\
\hline Muleta & $\begin{array}{l}\text { Su construcción se realiza según la solución de diferentes OBM. La funcionalidad } \\
\text { de este proyecto se basa en operar fraccionarios, decimales, porcentajes, horas y } \\
\text { minutos; según el movimiento del minutero y segundero se organiza la operación } \\
\text { a resolver. }\end{array}$ \\
\hline $\begin{array}{l}\text { Lotería } \\
\text { Matemática }\end{array}$ & $\begin{array}{l}\text { Es un juego que consiste en dividir un circulo en 6 partes, cada fracción lleva } \\
\text { un dibujo libre que representa diferentes números fraccionarios: p.ej. una casa } \\
\text { dividida en 14 cuadrados de los 14 solo 8 coloreados, lo cual sería igual a 8/14. } \\
\text { Luego de los dibujos se recortan 6 piezas que lleven el número de los fraccionarios } \\
\text { que se dibujaron. } \\
\text { El juego inicia con 2 a 4 jugadores los cuales poseen las fichas y deben ir } \\
\text { seleccionando la ficha que corresponde a cada fracción. El ganador es el que llena } \\
\text { la lotería de primeras. }\end{array}$ \\
\hline
\end{tabular}




\begin{tabular}{|l|l|}
\hline $\begin{array}{l}\text { Geometría de la } \\
\text { Multiplicación }\end{array}$ & $\begin{array}{l}\text { El objetivo es armar el resultado de una multiplicación a medida que se realiza una } \\
\text { figura geométrica sobre un círculo rodeado de los números de } 0 \text { a } 9 .\end{array}$ \\
\hline $\begin{array}{l}\text { Cilindro } \\
\text { Matemático }\end{array}$ & $\begin{array}{l}\text { La función del proyecto es girar las } 5 \text { tiras que se encuentran alrededor del rollo } \\
\text { hasta formar una operación, ya sea de suma, resta, división o multiplicación. Las } \\
\text { operaciones son de libre formulación para el docente. }\end{array}$ \\
\hline $\begin{array}{l}\text { Á } r \quad b \quad \text { o l I } \\
\text { Matemático }\end{array}$ & $\begin{array}{l}\text { Este proyecto consiste en ubicar unas tirillas de papel que tienen diferentes } \\
\text { números y signos matemáticos de operaciones. A medida que se desliza la tirilla } \\
\text { se forma un ejercicio con su respectiva respuesta. }\end{array}$ \\
\hline
\end{tabular}

\subsection{Competencias matemáticas y dificultades} de aprendizaje

La enseñanza de la matemática debe estar enfocada a la generación de destrezas para que el estudiante desarrolle la habilidad de solucionar problemas cotidianos, a la vez que se fortalece el pensamiento lógico y creativo (JiménezEspinosa, 2019). En Colombia, es el Ministerio de Educación Nacional (MEN, 2006), la entidad encargada de establecer los Estándares Básicos de Competencias en matemáticas, de las cuales se seleccionaron las que se indican en la tabla 2 , para este proyecto.

Tabla 2. Competencias matemáticas a ser apropiadas por los estudiantes.

\begin{tabular}{|l|l|l|l|l|}
\hline Competencia & \multicolumn{2}{|l|}{ Conceptual } & Lógico Matemática & Solución de OBM \\
\cline { 2 - 3 } & $\begin{array}{l}\text { ¿Qué es } \\
\text { Suma? }\end{array}$ & $\begin{array}{l}\text { ¿Qué es } \\
\text { multiplicación? }\end{array}$ & $\begin{array}{l}\text { Razonamiento a través de la } \\
\text { resolución de problemas }\end{array}$ & $\begin{array}{l}\text { Formulación de } \\
\text { ejercicios de suma, } \\
\end{array}$ \\
\cline { 2 - 3 } & $\begin{array}{l}\text { ¿Qué es } \\
\text { Resta? }\end{array}$ & $\begin{array}{l}\text { ¿Qué es } \\
\text { fraccionarios? }\end{array}$ & & $\begin{array}{l}\text { resta, división, } \\
\text { multiplicación, } \\
\text { fraccionarios. }\end{array}$ \\
\cline { 2 - 3 } & $\begin{array}{l}\text { ¿Qué es } \\
\text { división? }\end{array}$ & & & \\
\hline
\end{tabular}

García-Bustamante (2018), propone que en el aprendizaje de las operaciones directas, como la suma y la multiplicación, si no se guarda una relación estricta con el nivel mental, el ejercicio se convierte en adiestramiento puramente mecánico y memorístico. Esto deriva en un aprendizaje con enfoque tradicional, pero no presupone que los estudiantes tengan un pensamiento operatorio: es decir, se realizan las operaciones sin comprender su significado (Jiménez-Espinosa \& Sánchez-Bareño, 2019).

Sin embargo, cuando se habla de aprendizaje suelen aparecer diferentes obstáculos como las Dificultades de Aprendizaje en Matemáticas (DAM), lo cual suele constituir un desafío para entender todos los conceptos matemáticos, las bases del cálculo, el lenguaje de los símbolos matemáticos y la capacidad para resolver problemas matemáticos (García-Quiroga, Coronado \& Giraldo-Ospina, 2017; CastroRobles, Niño-Vega \& Fernández-Morales, 2020). En otras palabras: las matemáticas no son nada fáciles de aprender, su aprendizaje requiere la creación de significados abstractos, la codificación y descodificación de símbolos, y la capacidad de construir relaciones en el plano de lo posible (Dorado \& Díaz, 2014). 
Guerra-Guerra (2010), clasificó las DAM a través de 5 subtipos: discalculia verbal, discalculia ideognóstica, discalculia protagóstica, discalculia léxica y discalculia gráfica. El aprendizaje de las matemáticas es un aprendizaje complejo, y debe acompañarse de la maduración neurobiológica oportuna que permita alcanzar un nivel particular de desarrollo cognitivo, el cual a su vez sustenta los aprendizajes matemáticos (GonzálezNieves, Fernández-Morales \& Duarte, 2016).

\subsection{METODOLOGÍA}

La presente investigación tiene un enfoque mixto (Zuluaga-Duque, 2017), ya que, mediante el componente cuantitativo, se pretende medir el razonamiento lógico de los estudiantes que presentan dificultades cognitivas y metacognitivas en el área de matemáticas. Por medio del componente cualitativo, se espera observar el comportamiento y la actitud que asumen dichos estudiantes, frente a las actividades que se les presenta dentro y fuera del aula de clases.

El método en el cual se basa este estudio es de tipo inductivo, ya que tal y como lo afirma Ciro (2012), se hace una observación individual de los hechos o sucesos que enmarcan el problema de investigación. Posteriormente, se analizan cada una de las conductas y las características que se presentan en el campo o entorno en donde se desarrolla el estudio (Salcedo \& Fernández, 2018). El proceso finaliza con los resultados, luego de haber realizado comparaciones y experimentos antes, durante y después de haber intervenido la situación problémica (SilvaCañaveral, 2017).

En cuanto a la población objeto de estudio, en esta investigación se trabajó con 84 estudiantes de grado sexto, pertenecientes a la Institución Educativa Técnico Bellas Artes, de carácter público, ubicada en el municipio de Sogamoso, Boyacá. Esta institución ofrece servicios educativos de niveles de preescolar, básica primaria, secundaria y media, a estudiantes pertenecientes a estratos 1 y 2 de la población urbana del municipio. En cuanto a la muestra, se seleccionaron 23 de los 84 estudiantes, 11 hombres y 12 mujeres. Estos estudiantes fueron seleccionados debido a que presentaron DAM en la prueba Inicial.

La metodología se desarrolló en tres fases: en la primera, se presentó la estrategia $A B P$ y se aplicó la prueba diagnóstica a los estudiantes. En la segunda, se realizó la actividad de aula, para lo cual la muestra se dividió en dos grupos: uno que utilizó folletos y otro que usó un MEC, para adelantar el proyecto. En la tercera fase, se aplicó la prueba final y se analizó la información.

Las pruebas inicial y final se basaron en 30 preguntas, de las cuales 9 consistían en la solución de OBM, 7 en resolución de problemas y razonamiento lógico matemático, y 14 conceptuales y numéricas. Las pruebas se elaboraron de acuerdo a las 3 competencias de la tabla 1: conceptual, razonamiento y solución de OBM.

En la calificación se tuvo en cuenta una escala de valoración de: Bajo, Medio y alto. Los estudiantes que estaban en valoración de desempeño bajo, habían cometido entre 19 a 30 errores, Medio de 10 a 19 errores y alto entre 0 a 10 errores. El análisis de la información se realizó a través del software libre $\mathrm{R}$ (BarreraMesa, Fernández-Morales \& Duarte, 2018).

\section{RESULTADOS Y DISCUSIÓN}

\subsection{Actividad Inicial}

En primer lugar, se reunió a los 84 estudiantes de los grados sexto, con el fin de realizar un feedback entre el expositor y los estudiantes, para afianzar los conceptos. Luego se eligieron 10 estudiantes al azar, los cuales pasan a la tarima y en sus palabras explican los conceptos mencionados anteriormente. Para finalizar, tanto el público como los estudiantes seleccionados 
se indagan unos a otros sobre la temática, y expresan al expositor sus dudas.

El test inicial se aplicó de forma virtual, a través del software Kahoot, a la mitad del grupo, con una duración de 30 minutos. La otra mitad del grupo presentó la prueba de forma tradicional, ya que no había suficientes computadores con conectividad a internet.

El resultado del test inicial se observa en la figura 1. El $27 \%$ (23 estudiantes), figuran en el nivel de desempeño bajo; $66 \%$ (55 estudiantes), figuran en el nivel de desempeño básico; y $7 \%$ (6 estudiantes) demuestran nivel superior. Los 23 estudiantes del nivel bajo, fueron seleccionados como la muestra del presente estudio.

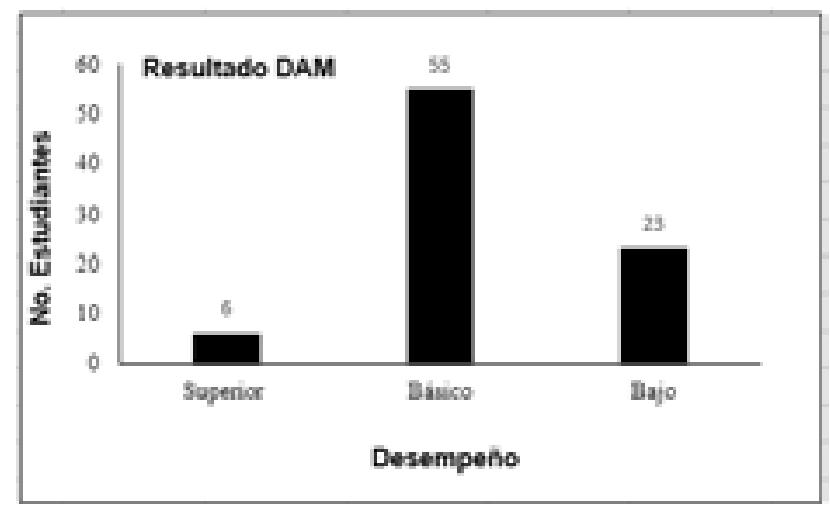

Figura 1. Resultados test inicial.

En cuanto a las competencias, se observa que la competencia en solución de OBM alcanza un $44 \%$, lo cual corresponde a 10 estudiantes; en la competencia conceptual de OBM, un $69 \%$ se encuentra en bajo, correspondiente a 16 estudiantes; y en la competencia de razonamiento se tiene un $87 \%$, correspondiente a 20 estudiantes. El anterior análisis se refiere a los 23 estudiantes que resultaron con menor desempeño en la aplicación del test inicial.

\subsection{Aplicación ABP en el aula}

La aplicación de la metodología ABP inició por la división de los 23 estudiantes en dos grupos: Uno construye los prototipos matemáticos propuestos a través de un folleto $(A)$, mientras que el otro a través de un MEC (B).

El grupo A se organiza por parejas y se les explica la dinámica del ejercicio. Luego, se inicia la resolución de las operaciones en forma tradicional sobre el folleto, y a medida que las van solucionando van construyendo el proyecto. Con el fin de afianzar los conocimientos previos y plasmarlos sobre la construcción que cada uno está realizando, el docente guía pasa continuamente por cada uno de los grupos, supervisando que el proceso se esté realizando de manera correcta, al igual que para resolver inquietudes sobre el tema.

Grupo A: se observó que los estudiantes en el transcurso de hora y media, indagaron y mostraron interés en solucionar cada una de las operaciones que se encontraban en los pasos de construcción. Esto con el fin de terminar el proyecto que les iba a facilitar su aprendizaje en las matemáticas. Cabe resaltar que se realizaron entre 5 y 6 preguntas por grupo. Solo un grupo tardó más del tiempo establecido, para el que se adicionaron 10 minutos y se finalizó por completo.

Grupo B: se estableció el mismo tiempo de construcción que para el grupo A. En este caso, el grupo logró terminar la construcción en el tiempo indicado y sin muchas dificultades, ya que el MEC que se estableció planteaba quices en forma de juegos interactivos. Se tuvo un promedio de 6 a 7 preguntas por grupo, lo que indica que la participación con esta metodología fue más activa.

La actividad de aula finalizó con una exposición en forma de feria, donde cada grupo explicaba a los demás el funcionamiento y la construcción del proyecto, al igual que la aplicabilidad en el aula de clases. Finalmente, cada estudiante se llevó su proyecto, para emplearlo en el transcurso de las clases de matemáticas. 
En general, el comportamiento de los estudiantes fue de concentración, causada por el interés de construir algo que les iba a ayudar a aprender matemáticas a través del juego y de nuevos métodos.

En la entrevista realizada a algunos estudiantes, se logró establecer que se sintieron felices en la construcción de los proyectos. Esto ayudó a que ellos se sintieran motivados y lograran planear, solucionar y finalizar los problemas que se formularon en la aplicación del ABP.

\subsection{Comparación pruebas inicial y final}

En el test final se evidenció una notable mejora en comparación del test inicial, lo cual indica que el método ABP a través de las TIC resulta ser eficaz para estudiantes que presentan DAM. En la figura 2 se muestra un paralelo entre los resultados iniciales y finales.

El test inicial ofrece un desempeño bajo con $27 \%$, básico con $66 \%$ y superior con $7 \%$. En el test final se observa el notable avance en la superación de dificultades matemáticas, ya que los estudiantes se encuentran en un nivel básico con un $17 \%$, y superior en el $83 \%$.

En el análisis estadístico conceptual de variables, se realizó la descripción de la población y la comparación de los resultados de las pruebas inicial y final. Estas pruebas se aplicaron antes y después de haber implementado el ambiente de aprendizaje basado en la solución de OBM,
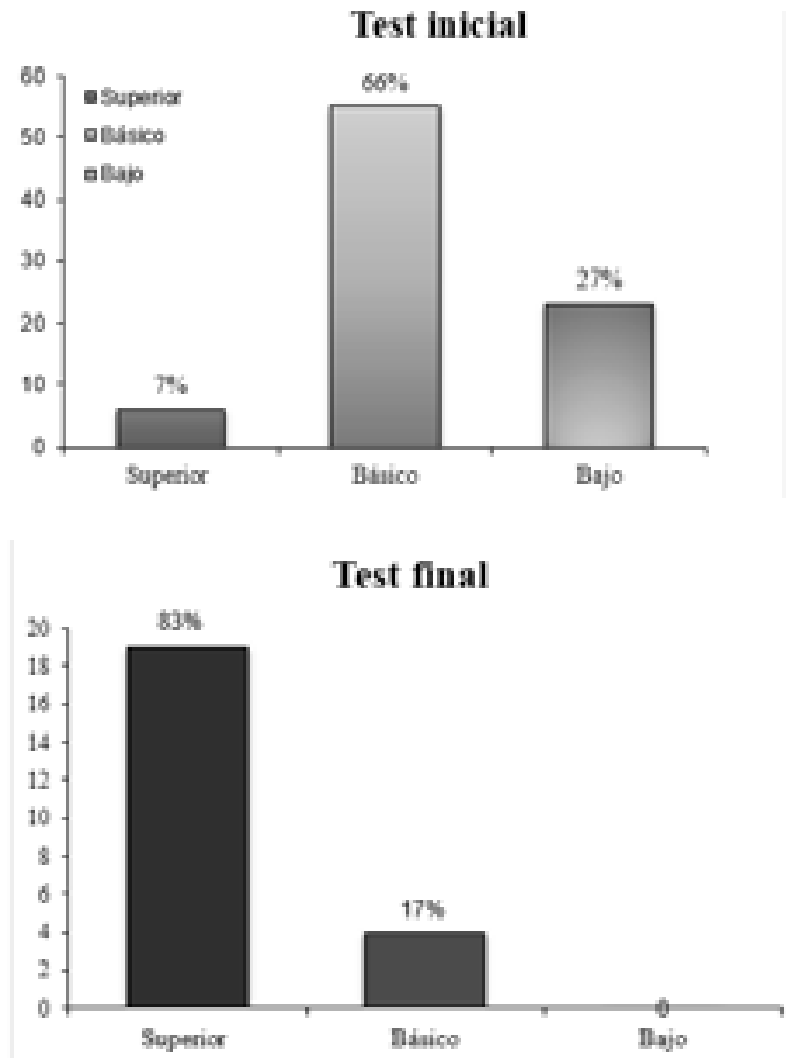

Figura 2. Comparación de pruebas inicial y final

a través de proyectos, por cada una de las competencias evaluadas.

El análisis implicó determinar el desempeño de las pruebas inicial y final. Luego se efectuó la comparación de medias por competencia evaluada, en las pruebas inicial y final, utilizando tablas de contingencia, basándose en un test estadístico y al contraste mediante la razón de verosimilitudes (Díaz, 2009).

En la tabla 3 se presenta un resumen del número de errores que se dieron en la prueba inicial.

Tabla 3. Número de errores test inicial.

Tabla 3. Número de errores test inicial.

\begin{tabular}{|c|c|c|c|c|c|c|c|c|}
\hline \multirow{2}{*}{$\bar{x}$} & \multirow{2}{*}{$s d$} & \multirow{2}{*}{$c v$} & \multirow{2}{*}{ Skewness } & \multirow{2}{*}{ Kurtosis } & \multicolumn{3}{|c|}{ Quantiles } & \multirow{2}{*}{$n$} \\
\hline & & & & & $Q_{1}$ & $Q_{2}$ & $Q_{3}$ & \\
\hline 20.57 & 1.56 & 7.59 & 1.25 & 4.17 & 19.50 & 20 & 21 & 23 \\
\hline
\end{tabular}


De la tabla3 se puede afirmar que, en promedio, los estudiantes cometen entre 20 a 21 errores en la prueba inicial. Igualmente, el $50 \%$ de los estudiantes comete 20 errores o menos y el otro $50 \%$ comete 20 errores o más. Debido a que el coeficiente de variación es menor que 30 , se puede afirmar que este conjunto de datos presenta baja variabilidad. Además, como el coeficiente de asimetría es mayor que cero, este conjunto de datos presenta una distribución asimétrica positiva, y la forma del histograma es leptocúrtica (presenta alto grado de concentración), puesto que el valor del coeficiente de curtosis o apuntamiento es mayor que 3. Los estudiantes cometieron con mayor frecuencia 19 a 21 errores. Esto corrobora el valor que se obtuvo con el coeficiente de asimetría de 1.56, lo cual ratifica que la variable número de errores en la prueba inicial tiene una distribución asimétrica positiva.

En la tabla 4 se presentan los resultados del test final, con la misma estructura de la tabla 3.

Tabla 4. Número de errores prueba final.

Tabla 4. Número de errores prueba final.

\begin{tabular}{|c|c|c|c|c|c|c|c|c|}
\hline \multirow{2}{*}{$\bar{x}$} & \multirow{2}{*}{$s d$} & \multirow{2}{*}{$c v$} & \multirow{2}{*}{ Skewness } & \multirow{2}{*}{ Kurtosis } & \multicolumn{3}{|c|}{ Quantiles } & \multirow{2}{*}{$n$} \\
\hline & & & & & $Q_{1}$ & $Q_{2}$ & $Q_{3}$ & \\
\hline 5.73 & 3.13 & 54.65 & -0.0059 & 1.50 & 3 & 5 & 8.5 & 23 \\
\hline
\end{tabular}

De la tabla 4 se puede afirmar que, en promedio, los estudiantes cometen entre $5 \circ 6$ errores en la prueba final. Igualmente, el $50 \%$ de los estudiantes comete 5 errores o menos y el otro $50 \%$ comete 5 errores o más. Debido a que el coeficiente de variación es mayor que 30 , se puede afirmar que este conjunto de datos presenta alta variabilidad o poca concentración. Además, como el coeficiente de asimetría es prácticamente cero, este conjunto de datos presenta una distribución simétrica y la forma del histograma es platicúrtica (presenta bajo grado de concentración), puesto que el valor del coeficiente de curtosis o apuntamiento es menor que 3.

El histograma permite evidenciar que el número de errores por estudiante, se redujo notablemente entre las pruebas inicial y final, con una variación de 8 a 10 y de 4 a 6 errores, respectivamente. El coeficiente asimétrico fue de -0.0059 , lo que confirma una variable con distribución simétrica, indicando la eficiencia del ABP para la superación de dificultades de aprendizaje matemático.

En la literatura se afirma que implementar el ABP en el aula de clases, resulta ser una estrategia pedagógica óptima para fortalecer el proceso de enseñanza-aprendizaje, debido a que el estudiante abstrae el conocimiento de forma autónoma, por medio de la teoríay y la experimentación (Rodríguez-Sandoval, VargasSolano \& Luna-Cortés, 2010; Niño-Vega, Fernández-Morales \& Duarte, 2019). Asimismo, gracias a la llegada de las TIC al campo de la educación, se han venido desarrollando diferentes escenarios y ambientes de aprendizaje ideales para la difusión del conocimiento. Uno de ellos son los Entornos Virtuales de Aprendizaje (EVA), los cuales propician el aprendizaje activo de los estudiantes, a través de la interacción del contenido en espacios digitales (Ciro, 2012).

Finalmente, se puede decir que, aunque las TIC provee a los usuarios de múltiples herramientas informáticas para la interacción y presentación 
del contenido, es indispensable que el docente diseñe y valide estrategias pedagógicas eficaces (Niño-Vega \& Fernández-Morales, 2019). Esto permitirá transformar a las herramientas informáticas, en un material educativo didáctico útil para la enseñanza-aprendizaje.

\section{CONCLUSIONES}

La formación de los estudiantes en cuanto a la conceptualización de un nuevo método de aprendizaje-enseñanza, basado en la construcción de proyectos tecnológicos y solución de problemas a través de test interactivos virtuales, permite tanto a los docentes como a los estudiantes integrar herramientas tecnológicas en sus prácticas educativas.

Los resultados del test inicial evidenciaron que 23 de los estudiantes, presentaron diferentes dificultades en la comprensión de Operaciones Básicas Matemáticas (OBM). El test integró contenidos basados en las competencias de: razonamiento lógico matemático, conceptual de OBM y competencias de solución de OBM. Esto permitió a los estudiantes demostrar la dificultad que presentaban y posteriormente dinamizar su proceso educativo, mediante la aplicación del Aprendizaje Basado en Problemas (ABP), para mejorar su aprendizaje.

Luego de implementar el $A B P$, se evidenció que la única diferencia entre el grupo que usó folletos y el que usó el Material Educativo Computarizado (MEC), fue el tiempo en la realización de los proyectos. Esto ya que el resultado de las pruebas, mostró la misma mejora en cuanto al nivel de dificultad presente en cada competencia. No obstante, la observación del comportamiento de los estudiantes al construir el proyecto, permitió identificar que el ABP con el folleto, aumentó la concentración de los estudiantes en cuanto a la competencia conceptual. En cambio, en cuanto al uso del computador, se denotó un mayor interés en la competencia de razonamiento lógico matemático, mientras que en la competencia de solución de OBM hubo igualdad. Esto permite concluir que el ABP brindó resultados satisfactorios para el aprendizaje de los estudiantes.

En síntesis, la investigación permitió confirmar que, gracias a las TIC y el ABP, los estudiantes aprenden de forma autónoma y logran enriquecer su conocimiento a través de la experimentación simulada. Así mismo, se demostró que el docente no solamente es un guía que está a disposición del estudiante para orientarlo, sino que juega un papel fundamental como motivador y creador de escenarios de aprendizaje.

\section{REFERENCIAS BIBLIOGRÁFICAS}

Aldana-Bermúdez, E., \& López-Mesa, J. (2016). Matemáticas para la diversidad: un estudio histórico, epistemológico, didáctico y cognitivo sobre perímetro y área. Revista de Investigación, Desarrollo e Innovación, 7 (1), 77-92. doi: http://dx.doi. org/10.19053/20278306.v7.n1.2016.5602

Alvis-Puentes, J. F., Aldana-Bermúdez, E., \& Caicedo-Zambrano, S. J. (2019). Los ambientes de aprendizaje reales como estrategia pedagógica para el desarrollo de competencias matemáticas en estudiantes de básica secundaria. Revista de Investigación, Desarrollo e Innovación, 10 (1), 135-148. doi: 10.19053/20278306. v10.n1.2019.10018

Barrera-Mesa, M., Fernández-Morales, F. H., \& Duarte, J. E. (2017). Aprendizaje basado en proyectos colaborativos mediados por TIC para el desarrollo de competencias en estadística. Saber, Ciencia y Libertad, 12 (2), 220-232. Recuperado de: http:// www.sabercienciaylibertad.org/ojs/index. $\mathrm{php} / \mathrm{scyl} / \mathrm{article} / \mathrm{view} / 247$ 
Barrera-Mesa, C. E., Fernández-Morales, F. H., \& Duarte, J. E. (2018). Validación de un ambiente de aprendizaje para la enseñanza de operadores mecánicos en educación básica. Revista Espacios, 39 (25), 2. Recuperado de: http://www.revistaespacios.com/ a18v39n25/18392502.html.

Botero, F. M., Rentería, L., \& Vergara, F. (2016). El aprendizaje de las operaciones básicas matemáticas en educación primaria, mediado por ambientes virtuales de aprendizaje: el caso de la IE Pascual Correa Flórez del municipio de Amagá, IE San Luis del municipio de San Luis y Centro Educativo Rural el Edén del municipio de Granada (Tesis de maestría). Universidad Pontificia Bolivariana. Recuperado de: https://repository.upb.edu.co/ bitstream/handle/20.500.11912/2601/ Traba o $\% 20$ de $\% 20$ grado- $\% 20$ L\%C3\%B3pez\%20Flor-\%20Renteria\%20 Lucero-20Vergara\%20Fabi\%C3\%A1n. pdf?sequence $=1$

Buitrago-Guzmán, S. (2014). Relación entre la convergencia de medios y la experiencia de usuario. Dos iniciativas creativas en Colombia. Revista De Investigación, Desarrollo e Innovación, 4(2), 79-86. doi: http://dx.doi. org/10.19053/20278306.2958

Cáceres, C. A., \& Amaya, D. (2016). Desarrollo e interacción de un laboratorio virtual asistido y controlado por PLC. Entre Ciencia e Ingeniería, 10(19), 9-15. Recuperado de: http://www.scielo.org.co/ scielo.php?script=sci_arttext\&pid=S1909$83672016000100002 \&$ Ing=es\&tlng=es.

Castro-Robles, A., Niño-Vega, J., \& FernándezMorales, F. (2020). El acoso escolar como oportunidad para mejorar la convivencia en el aula. Educación y Humanismo,
22(38), 1-13.Doi: https://doi.org/10.17081/ eduhum.22.38.3675

Ciro, C. (2012). Aprendizaje Basado en Proyectos (A.B.Pr) Como estrategia de Enseñanza y Aprendizaje en la Educación Básica y Media. Universidad Nacional de Colombia.

Cruz-Rojas, G. A., Molina-Blandón. M. A., \& Valdiri-Vinasco. V. (2019). Vigilancia tecnológica para la innovación educativa en el uso de bases de datos y plataformas de gestión de aprendizaje en la universidad del Valle, Colombia. Revista de Investigación, Desarrollo e Innovación, 9 (2), 303-317. doi: 10.19053/20278306. v9.n2.2019.9175

Dorado, I., \& Díaz, J. L. (2014). La matemática como herramienta de modelización para dar respuesta a situaciones problema. Acta Latinoamericana de Matemática Educativa, (pp. 1151-1159). México, DF: Comité Latinoamericano de Matemática Educativa.

Fernández, F. H., \& Duarte, J. E. (2013). El aprendizaje basado en problemas como estrategia para el desarrollo de competencias específicas en estudiantes de ingeniería. Formación universitaria, 6 (5), 29-38. Doi: https://dx.doi.org/10.4067/ S0718-5006201300050000

Fernández-Guerrero, M. M., \& González-Ferro, V. (2017). Enseñar, el arte de transformar y crecer. Saber, Ciencia y Libertad, 12 (2), 167-174. Recuperado de: http://www. sabercienciaylibertad.org/ojs/index.php/ scyl/article/view/242

García-Bustamante, L. A. (2018). Un descubrimiento trascendental en matemáticas. Revista Boletín Redipe, 4(7), 86-112. Recuperado de: https:// 
revista.redipe.org/index.php/1/article/ view/374

García-Martín, J., \& Pérez-Martínez, J. (2018). Aprendizaje basado en proyectos: método para el diseño de actividades. Revista Tecnología, Ciencia y Educación, 10. Recuperado de: https://tecnologiaciencia-educacion.com/index.php/TCE/ article/view/194

García-Quiroga, B., Coronado, A., \& GiraldoOspina, A. (2017). Implementación de un modelo teórico a Priori de competencia matemática asociado al aprendizaje de un objeto matemático. Revista de Investigación, Desarrollo e Innovación, 7(2), 301-315. doi: https://doi. org/10.19053/20278306.v7.n2.2017.6072

Garzón-Saladen, Á., \& Romero-González, Z. (2018). Los modelos pedagógicos y su relación con las concepciones del derecho: puntos de encuentro con la educación en derecho. Revista de Investigación, Desarrollo e Innovación, 8(2), 311-320. doi: https://doi.org/10.19053/20278306. v8.n2.2018.7968

Gomez- Pablos, V. B. (2018). El valor del aprendizaje basado en proyectos con tecnologías: análisis de prácticas de referencia (Tesis doctoral). Instituto Universitario de Ciencias de la Educación. Recuperado de: https://knowledgesociety. usal.es/sites/default/files/tesis/Tesis\%20 Doctoral\%20-\%20Vero\%CC\%81nica\%20 Basilotta\%20Go\%CC\%81mez-Pablos.pdf

González-Nieves, S., Fernández-Morales, F., \& Duarte, J. (2016). Memoria de trabajo y aprendizaje: Implicaciones para la educación. Saber, Ciencia YLibertad,11(2), 161-176. doi:http://dx.doi.org/10.22525/ sabcliber.2016v11n2.161176 aprendizajeen matemáticas, orientaciones prácticas para la intervención con niños con discalculia. Revista Digital Eduinnova, 27, 14-18. Recuperado de: http://www. eduinnova.es/dic2010/dic03.pdf

Gutiérrez-Rico, D., Almaraz-Rodríguez, O. D., \& Bocanegra-Vergara, N. (2019). Concepciones del docente en sus formas de percibir el ejercicio de la investigación desde su práctica. Revista de Investigación, Desarrollo e Innovación, 10 (1), 149-162. doi: 10.19053/20278306. v10.n1.2019.10019

Gutiérrez-Rodríguez, C. (2018). Fortalecimiento de las competencias de interpretación y solución de problemas mediante un entorno virtual de aprendizaje. Revista de Investigación, Desarrollo e Innovación, 8(2), 279-293. doi: https://doi. org/10.19053/20278306.v8.n2.2018.7170

Hernández-Suárez, C., Prada-Núñez, R., \& Gamboa-Suárez, A. (2017). Conocimiento y uso del lenguaje matemático en la formación inicial de docentes en matemáticas. Revista de Investigación, Desarrollo e Innovación, 7(2), 287-299. doi: https://doi.org/10.19053/20278306. v7.n2.2017.6071

Jiménez-Espinosa, A. (2019). La dinámica de la clase de matemáticas mediada por la comunicación. Revista de Investigación, Desarrollo e Innovación, 10 (1), 121134. doi: 10.19053/20278306.v10. n1.2019.10016

Jiménez-Espinosa, A., \& Sánchez-Bareño, D. M. (2019). La práctica pedagógica desde las situaciones a-didácticas en matemáticas. Revista de Investigación, Desarrollo e Innovación, 9 (2), 333-346. doi: 10.19053/20278306.v9.n2.2019.9179

Guerra-Guerra, M. (2010). Dificultades de 
Malpartida- Márquez, J. D. (2018). Efecto del aprendizaje basado en proyectos en el logro de habilidades intelectuales en estudiantes del curso de contabilidad superior en una universidad pública de la región Huánuco (Trabajo de pregrado). Recuperado de: http://repositorio.upch. edu.pe/bitstream/handle/upch/1515/ Efecto_MalpartidaMarquez_Jose. pdf?sequence $=1$ \&isAllowed $=y$

Marino-Vera, H., Mendoza, L., \& GualdrónGuerrero, O. (2017). Medición automática de variables antropométricas para la evaluación de la respiración usando visión artificial. Revista de Investigación, Desarrollo e Innovación, 8(1), 161-169. doi: https://doi.org/10.19053/20278306. v8.n1.2017.7407

Martínez-López, L. G., \& Gualdrón-Pinto, E. (2018). Fortalecimiento del pensamiento variacional a través de una intervención mediada con TIC en estudiantes de grado noveno. Revista de Investigación, Desarrollo e Innovación, 9 (1), 91-102. doi: https://doi.org/10.19053/20278306. v9.n1.2018.8156

Ministerio de Educación Nacional, MEN. (2006). Estándares Básicos de Competencias en Matemáticas. Bogotá, Colombia.

Morales-Bueno, P. (2018). Aprendizaje basado en problemas (ABP) y habilidades de pensamiento crítico ¿una relación vinculante?. Revista Electrónica Interuniversitaria de Formmación del Profesorado, 21 (2), 91-108.

Niño-Vega, J. E., Martínez-Díaz, L. Y., Fernández-Morales, F. H., Duarte, J. E., Reyes-Caballero, F., \& Gutiérrez-Barrios, G. J. (2017). Entorno de aprendizaje para la enseñanza de programación en Arduino mediado por una mano robótica didáctica.
Revista Espacios, 38 (60), 23. Recuperado de: http://www.revistaespacios.com/ a17v38n60/17386023.html

Niño-Vega, J. A., Fernández-Morales, F. H., \& Duarte, J. E. (2019). Diseño de un recurso educativo digital para fomentar el uso racional de la energía eléctrica en comunidades rurales. Saber, Ciencia y Libertad, 14(2) 256-272. Doi: https://doi.org/10.18041/2382-3240/ saber.2019v14n2.5889

Niño-Vega, J. A., \& Fernández-Morales, F. H. (2019). Una mirada a la enseñanza de conceptos científicos y tecnológicos a través del material didáctico utilizado. Revista Espacios, 40 (15), 4. Recuperado de: http://www.revistaespacios.com/ a19v40n15/19401504.html

Ordóñez-Ortega, O., Gualdrón-Pinto, E., \& Amaya-Franky, G. (2019). Pensamiento variacional mediado con baldosas algebraicas y manipuladores virtuales. Revista de Investigación, Desarrollo e Innovación, 9 (2), 347-362. doi: 10.19053/20278306.v9.n2.2019.9180

Revelo-Rosero, J. E., Lozano, E., \& BastidasRomo, P. (2019). La competencia digital docente y su impacto en el proceso de enseñanza-aprendizaje de la matemática. Espirales: Revista Multidisciplinaria de investigación, 3 (28). doi: https://doi. org/10.31876/er.v3i28.630

Ruiz-Macías, E., \& Duarte, J. E. (2018). Diseño de un material didáctico computarizado para la enseñanza de oscilaciones y ondas, a partir del estilo de aprendizaje de los estudiantes. Revista de Investigación, Desarrollo e Innovación, 8 (2), 295-309. doi: https://doi.org/10.19053/20278306. v8.n2.2018.7966 
Rodríguez-Sandoval, E., Vargas-Solano, É., \& Luna-Cortés, J. (2010). Evaluación de la estrategia "aprendizaje basado en proyectos”. Educación y Educadores, 13(1). Recuperado de: https:// educacionyeducadores.unisabana.edu. co/index.php/eye/article/view/1618/2128

Salcedo-Ramírez, R. Y., Fernández-Morales, F. H., \& Duarte, J. E. (2017). Unidad didáctica para la enseñanza de probabilidad mediada por un OVA, orientada a un colegio rural del municipio de Paipa. Revista Colombiana de Tecnologías de Avanzada, 2 (30), 1-10. doi: https://doi.org/10.24054/16927257. v30.n30.2017.2739

Salcedo, S. P., \& Fernández, F. (2018). Mejoramiento de la escritura en inglés como lengua extranjera en niños con síndrome de Down mediada por las TIC. Revista Boletín Redipe, 6(11), 76-85. Recuperado de: https://revista.redipe.org/ index.php/1/article/view/402

Silva-Cañaveral, S. (2016). La investigacióncreación en el contexto de la formación doctoral en diseño y creación en Colombia. Revista de Investigación, Desarrollo e Innovación, 7(1), 49-61. doi: https://doi. org/10.19053/20278306.v7.n1.2016.5601

Trujillo-Losada, M. F., Hurtado-Zúñiga, M. C., \& Pérez-Paredes, M. J. (2019). Fortalecimiento de los proyectos educativos de las instituciones educativas oficiales del municipio de Santiago de Cali. Revista de Investigación, Desarrollo e Innovación, 9 (2), 319-331. doi: 10.19053/20278306.v9.n2.2019.9177

Vega-Monsalve, N. C., \& Ruiz-Restrepo, A. M. (2018). Retos y desafíos de la educación superior para responder a la demanda de profesionales en la subregión del Oriente Antioqueño Colombiano. Revista de Investigación, Desarrollo e Innovación, 9 (1), 115-126. doi: https://doi. org/10.19053/20278306.v9.n1.2018.8510

Vergara-Arrieta, J. J. (2015). Los sistemas de medición del desempeño estratégico frente a la competitividad y sustentabilidad. Saber, Ciencia y Libertad, 10 (2), 17-26. Recuperado de: https://dialnet.unirioja.es/ servlet/articulo?codigo $=5295019$

Vesga-Bravo, G. J., \& Escobar-Sánchez, R. E. (2018). Trabajo en solución de problemas matemáticos y su efecto sobre las creencias de estudiantes de básica secundaria. Revista de Investigación, Desarrollo e Innovación, 9 (1), 103-114. doi: https://doi.org/10.19053/20278306. v9.n1.2018.8270

Villamil-Rincón, D., Aldana-Bermúdez, E., \& Wagner-Osorio, G. (2018). Análisis de contenido del concepto de área en educación superior. Revista de Investigación, Desarrollo e Innovación, 8(2), 265-278. doi: https://doi. org/10.19053/20278306.v8.n2.2018.7964

Zuluaga-Duque, J. F. (2017). Relación entre conocimientos, saberes y valores: un afán por legitimar los saberes más allá de las ciencias. Revista de Investigación, Desarrollo e Innovación, 8(1), 61-76. doi: 10.19053/20278306.v8.n1.2017.5973 